\title{
ASIC Framework Simplified and Operationalised - An Operational Matrix for Optimising the Use of Technologies and Innovations in Medical Education
}

\author{
Joshua Owolabi (D) \\ Anatomy Department, Division of Basic Medical Sciences, University of Global Health Equity, Butaro, Rwanda \\ Correspondence: Joshua Owolabi, Email jowolabi@ughe.org
}

\begin{abstract}
The ASIC [adaptation, standardisation, integration and compliance] framework was developed to set the standard for the use of innovations and technologies in medical education. There is a need to develop frameworks and reference guides for educational technologies [EdTechs] and innovations, noting that EdTechs are becoming increasingly important to the delivery of medical education. The ASIC framework as previously published presents four major tenets including: adaptation, standardisation, integration and compliance. The operational matrix is now developed and presented in this article. Each tenet of the ASIC framework has key requirements or questions that the user of an EdTech or educational innovation should address. Each question represents a key requirement to be satisfied by the user of the EdTech to satisfy the ASIC tenets. Each question is also equally weighted as every other one. The matrix has 12 key questions, representing 12 key requirements. The results measurement can either be a fraction of 12 or a percentage. As a requirement, the minimum score under each category is 2 out of 3; ideally, optimisation of an educational innovation or technology requires that all boxes are checked in the affirmative. However, a minimum of 2 out of 3 in every category would indicate a minimum score or threshold. This effort is in line with previous and ongoing efforts to ensure that educational technologies and innovation that are deployed to deliver medical education are adapted for optimal performance, standardised for the use of training, integrated into the medical education delivery system, and compliant with professional, institutional, and regulatory standards.
\end{abstract}

Keywords: innovation, technology, medical education, ASIC, framework, operational matrix, adaptation, standardisation, integration, compliance

\section{Introduction}

The ASIC framework was recently published, as a framework that can help to optimize the introduction and use of innovations and educational technology in the delivery of medical education. ${ }^{1}$ A major consideration that warranted the development of the framework is the fact that innovations and educational technologies are becoming increasingly important and integral the delivery of medical education, especially Anatomy. ${ }^{2-6}$ For example, functional and clinical anatomy requires extensive exploration of the human body including its systems and their functions, hence, EdTechs in certain instances have been able to combine quality digital representation of the human body with simulations of functional phenomena, structural aberrations and procedures of interventions. Furthermore, EdTechs and educational innovations are becoming more diverse while the degrees of sophistication of the innovations and technologies have also increased; a situation that could become increasingly complex or even complicated. Consequently, the types of innovations and technologies that are being used could vary greatly from place to place. In addition, the manner in which such innovations and technologies are being used could also greatly vary based on a number of factors that might include educators and stakeholders' interests in technology, and the prevalent teaching and learning culture. ${ }^{7}$ Other influencing factors could include the type of curriculum being operationalized, the time allocated to the use of technology, acceptability of technology by learners, educators and other stakeholders within educational systems, the main regulatory 


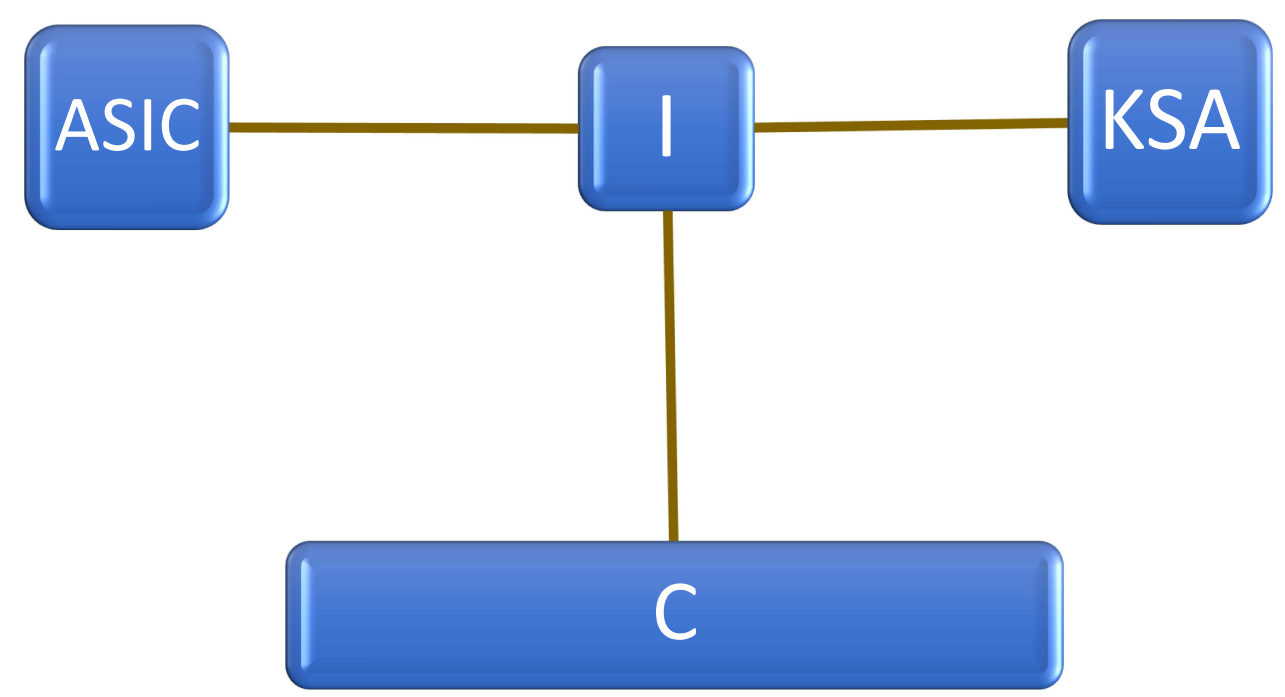

Figure I ASIC Simplified- the ASIC [Adaptation, Standardisation, Integration, and Compliance] framework linked to learning domains [Knowledge, Skill, Attitude] and curricular compliance [C]; the centre of integration, I, is always the context and space of learning such as the classroom, laboratory, simulation facilitates, hospital ward, the community.

Abbreviations: ASIC, Adaptation, Standardisation, Integration, Compliance; I, Integration Context or Setting eg Classroom, Laboratory, Simulation Facility etc; KSA, Knowledge, Skill, Attitude.

policies that help to oversee the delivery of medical education, institutional policies and practices as well as any other factor that could influence the use of innovations and technology at a particular point in time.

Another factor that has been identified might include economic factors such as the capacity to procure quality resources and to provide adequate infrastructural support that will serve as innovations and educational technologies. ${ }^{8}$ Beyond just the training, it has been previously reported that inequalities and inequities might limit the benefits of technological innovation in the healthcare systems. ${ }^{9}$ These realities might therefore imply that well-resourced institutions might have greater capacity to procure quality EdTechs and resources as well as the ability to train the users and equip them with adequate skills. Under resourced institutions on the other hand might not have such capacities. This explains why there might be a link between innovative technologies and social inequalities in health. ${ }^{10}$ Another major consideration is the state of the tech culture in an institution, and this often has to do with the level of appreciation for technology that the key stakeholders in the institution might have. This, for example, can influence their decisions and positions on the extent of the use of innovative approaches and technologies to support or deliver medical education. Paradigm could also play vital roles. For example, the older generation of educators might not have come to fully appreciate the significance of technology, not just to support the delivery of medical education currently, but the roles that such innovations and technologies might play in the future, and the need to prepare for such a future by integrating a tech culture into medical education and other aspects of education in general.

With all these considerations in mind as highlighted above, it is apparent that efforts should be invested into not just the acquisition of technologies or development of such technologies but to deploy such technologies and innovations in manners that will be optimally beneficial to learners and trainees. The ASIC framework was therefore designed and developed to serve such a purpose [Figure 1]. It is arguably the known foremost framework serving such purposes currently. It is believed that this framework could help to properly introduce innovations and medical education technologies into existing structures while at the same time it could help users of technology to ensure a structured approach to deploying technology in almost any context. The four key tenets of the framework include: Adaptation, Standardization, Integration, and Compliance. In the original publication of the ASIC framework, each of the tenets was broken down with explanations provided regarding what should be achieved to address each of these key tenets. ${ }^{1}$ In this particular effort, an operational matrix is being designed and developed to help users of the ASIC framework to operationalise the use of the framework while deploying innovations and educational technologies. It also helps to 


\begin{tabular}{|c|c|}
\hline A-Adaptation & dardisation \\
\hline $\begin{array}{l}\text { A1. Time allocation and specific duration of time for } \\
\text { use - specified time/period/duration for use [P] } \\
\text { A2. Specific objective identified- objective statement } \\
\text { [P] } \\
\text { AYes__ } \\
\text { Aearning outcome identified [P] }\end{array}$ & $\begin{array}{l}\text { S1. Time allocation on curriculum/syllabi- time/period } \\
\text { allocated }[\mathrm{P}] \\
\text { S2. Method of use is pre-determined and consistent- } \\
\text { method and protocol guide [P] } \\
\text { S3. Alignment with specific pedagogy or teaching } \\
\text { method- specific pedagogy or learning theory aligned } \\
{[\mathrm{P}]}\end{array}$ \\
\hline I- In & $\mathrm{C}-\mathrm{Cc}$ \\
\hline 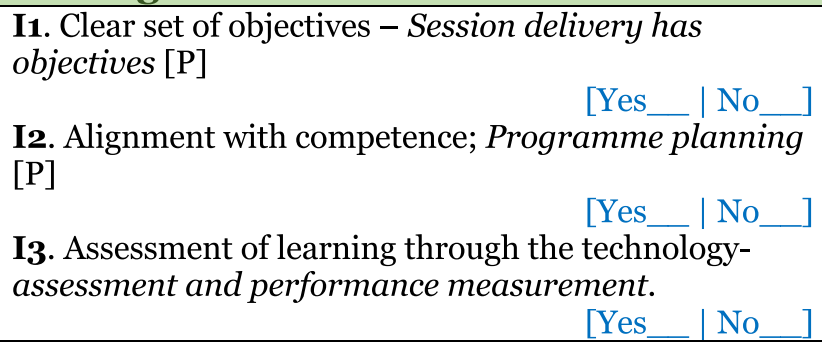 & $\begin{array}{l}\text { C1. Supported by institutional policies- institutional } \\
\text { compliance }[\mathrm{P}] \\
\text { C2. Supported by regulatory policies- regulatory } \\
\text { compliance }[\mathrm{P}] \\
\text { C3. Supported by learning culture- cultural } \\
\text { compliance }[\mathrm{P}]\end{array}$ \\
\hline
\end{tabular}

Figure 2 ASIC Framework Operational Matrix. ASIC Framework Operational Matrix has specific considerations under the four tenets of ASIC. Each of the tenets has 3 questions that validate it [eg Al= Adaptation-related question I; $\mathrm{C} 3=$ Compliance-related question 3]. The user is required to confirm each consideration [Yes] or decline [No]. Each response has an attached value. Each consideration under each ASIC tenet is coded and a specific score is allocated to it. Practically, there are overall 12 considerations that serve as the overall indicators of the operational performance level of an educational innovation or technology based on the ASIC Framework. The final outcome can be presented simply as a whole number and measured as a fraction of 12 , eg $10 / 12$. Alternatively, the score can be computed as a percentage to provide a more practical proportional representation of operational performance.

Abbreviations: A, Adaptation; S, Standardisation; I, Integration; C, Compliance; P, Prompt.

measure the level of performance or potential performance of such technologies, hence, measuring the degree of success deployment and consequently, optimisation of innovations and educational technologies.

\section{The ASIC Framework Operational Matrix [Figure 2]}

The ASIC Framework could help to optimise the use of an educational technology or innovation. It demands that the key competences $[\mathrm{C}]$ that an EdTech or innovation helps to achieve should be clearly identified. It further requires that these competences should be linked to learning in one or more basic domains ie KSA- Knowledge, Skill, Attitude. The practical use of this framework could be highlighted as follows:

1. Use the framework for the justification of a technology or innovation to deliver medical education.

2. Identify key competences to accomplish.

3. Set up integrating centre and deliver optimally.

4. Identify, accomplish, and measure learning in the 1-3 basic domains.

The matrix helps to operationalise the framework. The framework has specific questions that address the key considerations that underscore how effectively an educational technology or innovation is being deployed [or planned] to support teaching and training. Each ASIC tenet has three key questions associated with it, and to which the user of the framework can respond Yes/No. A yes earns a full score of 1 for each question and a No is scored a 0 . To help the user, each question has a prompt. There are 12 prompts altogether. The framework can be used manually on paper or digitally. In the paper version [as published in this article], the user can look up the prompt in order to be sure of which response to provide, hence ensuring the accuracy of responses. In the digital version [yet to be published], the user can simply click on the prompt $[\mathrm{P}]$ to bring up the prompts. Also, the scores are automatically recorded and presented via a dynamic digital 

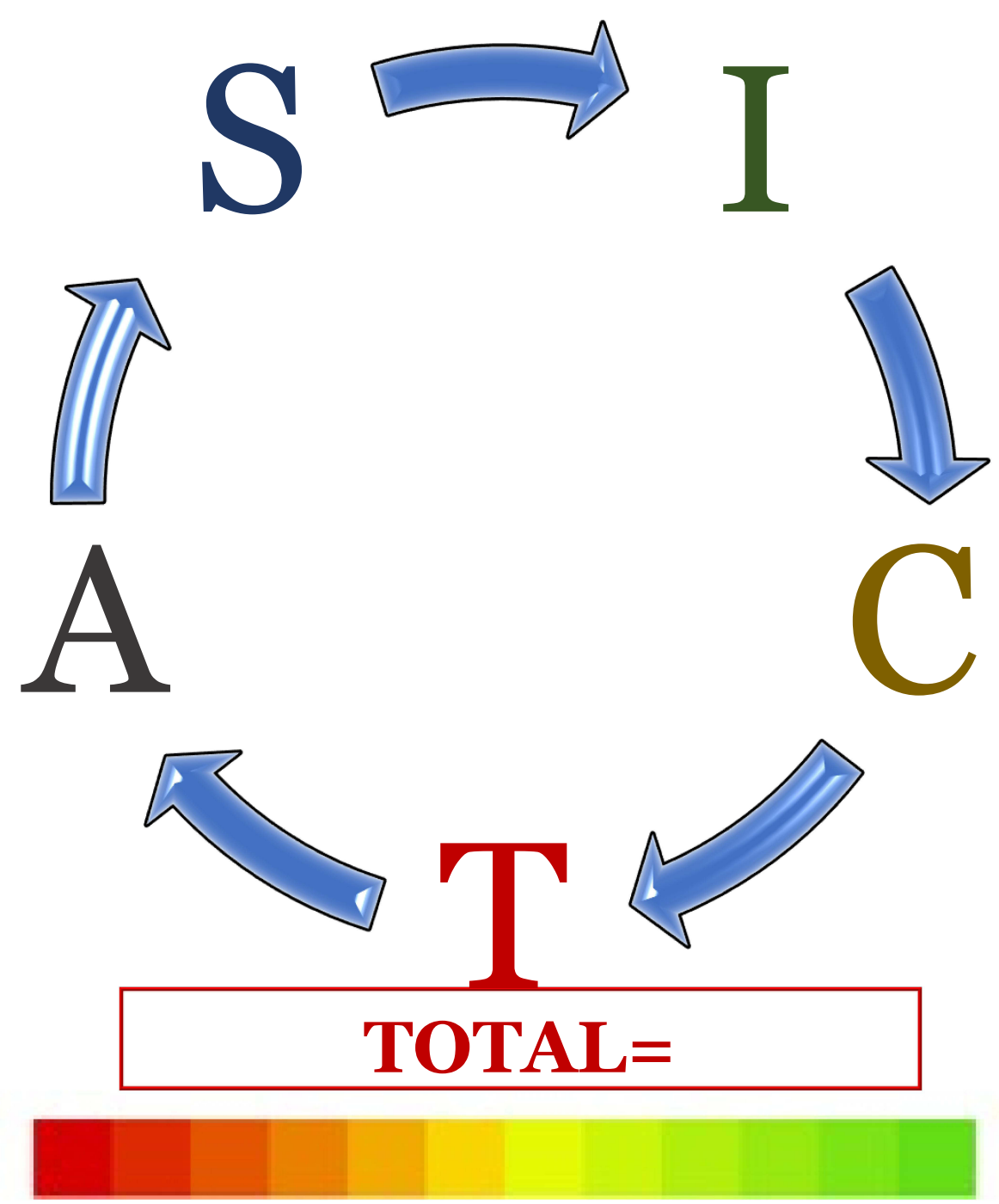

Figure 3 ASIC Framework Matrix digital result interface. This serves the purpose of a dynamic interface to present the measurements of the matrix in a way that practically helps to appreciate the potential performance of the educational technology or innovations being considered.

Abbreviations: A, Adaptation; S, Standardisation; I, Integration; C, Compliance.

interface in the digital version [Figure 3]; whereas in the manual/paper version, the user is required to manually record the score and compute the final score manually. Also, a worksheet [Figure 4] is prepared for planning/evaluation purposes. To use the worksheet, the user(s), who might be a faculty member or members of an academic committee, might simply work with the worksheet. Using the prompts as a guide, the worksheet is used to reflect, formulate, and provide specific responses to the requirement under each ASIC tenet. Ability to provide answers to all the requirements would be an indication of optimal deployment of a specific technology or innovation. It should be noted that this provides measurements as such for each technology, not an entire educational system set-up.

\section{Prompts}

\section{A- Adaptation}

A1: Do you have time allocated for the use of this technology on your syllabus and/or timetable?

A1 [Alternative]: Did you plan and weighed appropriately the duration of use of the technology?

A2: Can you indicate at least 1 objective that this technology helps you to achieve in connection with your session and/or programme objective? 


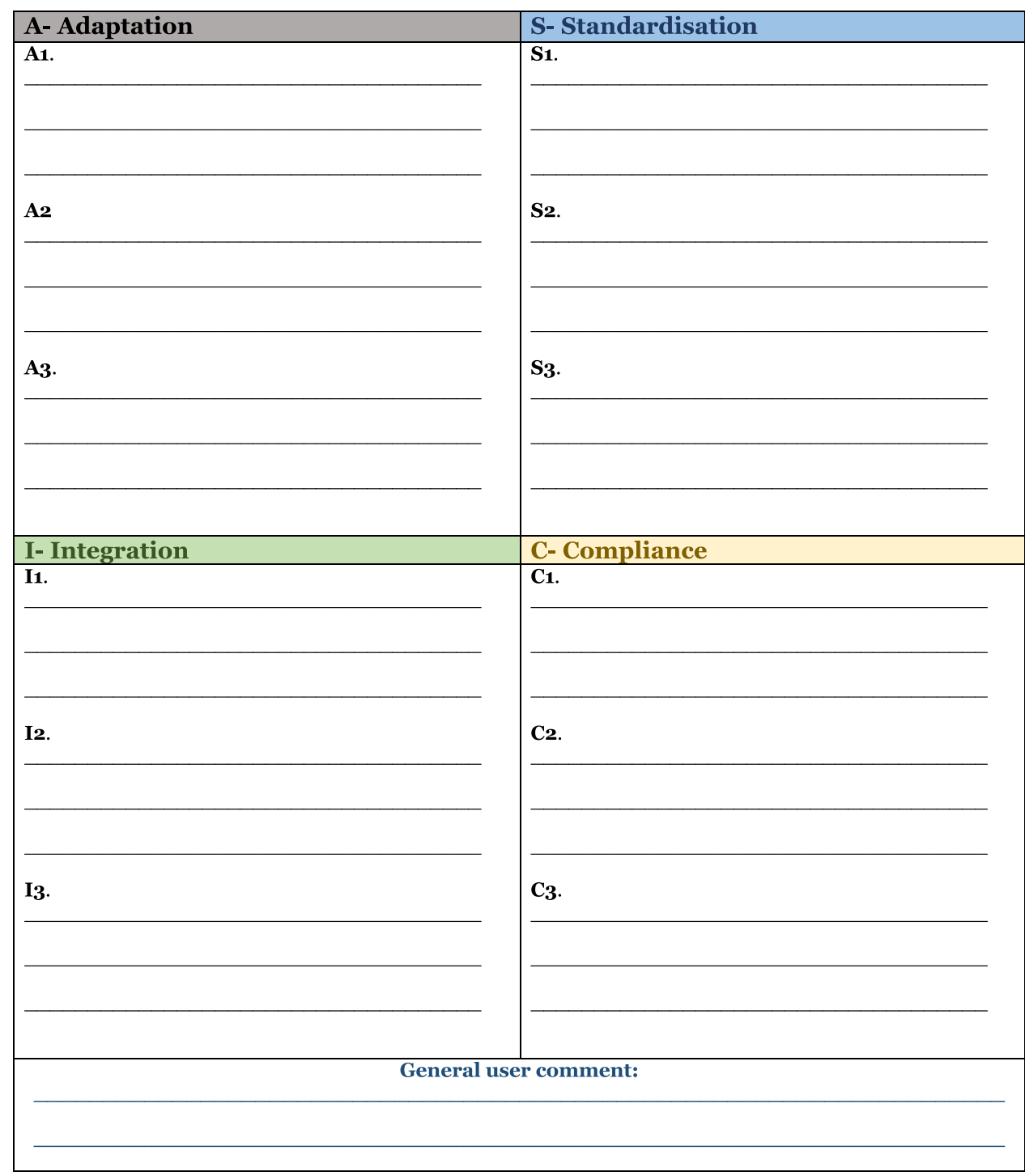

Figure 4 ASIC Framework Matrix Worksheet. A worksheet that could help to assess, measure, and document the optimization of medical education technologies and innovations through the operationalization of the ASIC Framework. Using the prompts under each ASIC tenet, educator or institution can reflect and provide concise and concrete answer to each question/requirement.

Abbreviations: A, Adaptation; S, Standardisation; I, Integration; C, Compliance.

A3: Can you indicate at least 1 learning outcome that this technology helps you to achieve in connection with your programme objective and/or competency?

\section{S- Standardisation}

S1: Is there time allocation or unit allocation for the use of the technology in your curriculum?

S2: Do you have a standard and consistent way of using this technology in alignment with course objectives/Do you have a protocol for the use of this technology?

S3: Is your use of this educational technology/ innovation aligned with any specific pedagogy or a learning theory [eg Medical simulation, digital dissection, case based collaborative learning | Constructivism, Connectivism etc.]?

\section{I- Integration}

I1: Did you formulate objectives for the use of the technology? 
I3: Can you identify at least 1 competency in each KSA domain that the use of the technology for your programme [not just course] can help to achieve?

I3: Is the use of the technology in your course/session specifically assessed ie included in the formative or summative assessments?

\section{C- Compliance}

$\mathrm{C} 1$ : Is your use of this technology supported by at least 1 specific policy in your institution as a marker of institutional compliance?

$\mathrm{C} 2$ : Is your use of this technology supported by at least 1 specific policy by your programme regulator as a marker of regulatory compliance $[\mathrm{P}]$

C3: Is your use of this technology supported by the teaching-learning culture of your programme/institution as a marker of cultural compliance $[\mathrm{P}]$

In the digitised version of the matrix [which is being developed as a software], each response to the consideration, under an ASIC tenet is calculated and the total is presented as a percentage which is an indication of the operational performance of technology. Another feature of the digitalized version of the matrix is the presentation of the matrix measurements in a graphical form that shows scores per matrix, colour codes each tenet based on performance and presents the overall performance on a colour coded scale bar. This is to give a visual representation of the performance of the innovation of educational technology being measured based on the ASIC Framework. In addition, a failure in any aspect of the tenet is coloured red. The overall measure is presented and accompanied with a comment that could also flag any aspect that requires further consideration to enhance performance. Very importantly, a red colour in an ASIC aspect would mean that the tool is failing under the specific ASIC tenets and as a rule of the thumb, the tool is failing operationality test. The solution is to make corrections to the problem[s] and reuse the matrix again.

\section{Guiding Rules}

1. Optimal performance requires an average of a score of 2.5-3 across all ASIC aspects.

2. Minimal score of 2 is required in all ASIC aspect.

3. Score measures potential of operationality; which is achieved when the innovation or technology in question is optimally used as indicated.

4. Measurement failure, under any of the ASIC tenets/area would mean poor operationality and performance measurements, even with a good overall score; consequently, the area of failure should be addressed until a "pass" score is attained in the category.

5. The pass score for an ASIC tenet is $2 / 3$ or 0.7 .

6. The overall pass score is $2 / 3$ or $\sim 70 \%$; as an average score across all ASIC areas.

\section{ASIC Framework Matrix Worksheet- Based on the Matrix Prompts}

The worksheet serves as an extended version of the operationalizing matrix which could help to assess, measure, and document the optimization of medical education technologies and innovations through the operationalization of the ASIC Framework further extensively. In this instance, the worksheet can help individual medical educators, medical education planning or reform committees and other institutional departments to measure how valuable educational technologies and innovations could be to the delivery of medical education. It could serve both an immediate measurement tool as well as a tool for reflective practice in the context of using medical education technologies and innovations.

\section{Guide for the Use of the ASIC Framework Matrix Worksheet}

1. Consider each ASIC Framework tenet

2. Read each prompt under each ASIC tenet

3. Reflect on the answer to each prompt and formulate a concise and accurate response

4. Record the response against the ASIC Tenet number eg AP1

5. If able to provide a response; a score of 1 [one] allocated 
6. If no answer is applicable; a score of 0 [zero] is recorded

7. For any question with a score of 0 [as in no. 6 above]; record this ASIC tenet number as a "requirement to fulfil $[R F] "$ during a next review. This is important to improvement and evaluation.

\section{Moving Forward}

There is continuous effort to conduct usability texts with this framework, particularly under various curricular types of model and educational context and cultures. This would be a way to ensure that the ASIC instruments would adequately meet the need of stakeholders in the medical education and training institutions including students and trainees, educators, and academic leaders. There is always a need to put to test new and emerging educational innovations and innovative approaches to teaching and training; ${ }^{11}$ so also, the frameworks and other validation and evaluation tools. Efforts such as this should continue as there is a need for significant investment in research and product development to support the development and the integration of technologies into medical education and healthcare. The need to build human capacity in the relevant sectors to these effects have been previously highlighted. ${ }^{12}$

It is equally important to highlight the benefits of standardizing the use of EdTechs and innovations to support medical education to students, trainees and learners. Clearly, consistency and methodical deployment of EdTechs and innovation would help to ensure standard practices about the use of such technologies and innovations. Medical education is clearly highly regimented, structured, and standardized, hence, applying these same principles to the use of EdTechs and innovations is equally an important consideration. Consequently, a way to ensure that such best practices are enshrined into the culture of training and learning in medical and allied health institutions is to provide a framework such as the ASIC Framework as well as complementary implementation tool such as its operational matrix to implement such standard and best practices.

It would be important to further advocate for more integral roles of educators in the conception of ideas, design, development, and the deployment strategies to ensure optimal use of educational technologies and innovations. There is a lot more that educators can do in this regard. It is also vital to appreciate the fact that innovations and technologies are becoming increasingly important to the delivery of health services and advancements through research and education. One thing that needs to be emphasised along these lines of development is the need to promote and enshrine a culture of technology use and innovativeness in the systems and institutions that train medical and health workers. Technology, rather than being seen as an optional and complementary tool should be considered an integral tool for cutting edge research and service delivery. Considering these realities, development of tools and instruments, such as the ASIC Framework and its operational matrix [Figure 2] as well as other strategies to adapt, integrate, standardise, and ensure the best use of innovations and technology in compliance with standard and best practices would significantly contribute to this effort. There is definitely a need to train more tech competent and compliant physicians. ${ }^{13,14}$ This must start with the educators and medical education stakeholders. There is a need to ensure a tech-supportive culture. Artificial intelligence is currently deployed and promoted in several fields of endeavours because of its immense potentials to shape the ways of life in the nearest future- in all walks of life. Unfortunately, the health care system is yet to adequately grasp this reality and to appreciate the potentials of certain technologies and innovations such as artificial intelligence or AI. ${ }^{15-18}$ This is just one of the several instances whereby a tech-supported medical education system could be revolutionary. It is a way of preparing for the future of healthcare service delivery.

\section{Conclusion}

The ASIC framework operational matrix is hereby developed for optimising the use of educational technologies [EdTechs] and innovations in medical education.

This is to ensure that educational technologies and innovation are deployed to deliver medical education in line with identified standards such that they are adapted for optimal performance, standardised for the use of training, integrated into the medical education delivery system, and compliant with professional, institutional, and regulatory standards.

\section{Disclosure}

The author reports no conflicts of interest in this work. 


\section{References}

1. Owolabi J. Proposing a framework guide for the integration of educational technologies and innovations into the teaching of anatomy and medical sciences: the ASIC framework. Adv Med Educ Pract. 2021;12:1277-1282. doi:10.2147/AMEP.S338262

2. Fallavollita P. Innovative technologies for medical education, human anatomy - reviews and medical advances, Alina Maria Sisu. IntechOpen. 2017. doi:10.5772/intechopen.68775

3. Santana EA, Orquera PA, Valenzuela JJ, Orellana MI, Gold MH, De La Paz Garcia G. Anatomical software as a tool in the teaching-learning process of human anatomy. Literature review. FASEB J. 2020;34(S1):1. doi:10.1096/fasebj.2020.34.s1.09262

4. Zargaran A, Turki MA, Bhaskar J, Spiers H, Zargaran D. The role of technology in anatomy teaching: striking the right balance. Adv Med Educ Pract. 2020;11:259-266. doi:10.2147/AMEP.S240150

5. Zhao J, Xu X, Jiang H, et al. The effectiveness of virtual reality-based technology on anatomy teaching: a meta-analysis of randomized controlled studies. BMC Med Educ. 2020;20:127. doi:10.1186/s12909-020-1994-z

6. Dawidziuk A, Kawka M, Szyszka B, Wadunde I, Ghimire A. Global access to technology-enhanced medical education during the COVID-19 pandemic: the role of students in narrowing the gap. Glob Health Sci Pract. 2021;9(1):10-14. doi:10.9745/GHSP-D-20-00455

7. Owolabi J, Bekele A. Implementation of innovative educational technologies in teaching of anatomy and basic medical sciences during the COVID-19 pandemic in a developing country: the COVID-19 silver lining? Adv Med Educ Pract. 2021;12:619-625. PMID: 34135653; PMCID: PMC8197662. doi:10.2147/AMEP.S295239

8. Millan J, Yunda L, Valencia A. Analysis of economic and business factors influencing disruptive innovation in telehealth. NOVA. 2017;15(28):125136. doi: $10.22490 / 24629448.2136$

9. Glied S, Lleras-Muney A. Technological innovation and inequality in health. Demography. 2008;45(3):741-761. doi:10.1353/dem.0.0017

10. Weiss D, Rydland HT, Øversveen E, Jensen MR, Solhaug S, Krokstad S. Innovative technologies and social inequalities in health: a scoping review of the literature. PLoS One. 2018;13(4):e0195447. doi:10.1371/journal.pone.0195447

11. Rohlfsen CJ, Sayles H, Moore GF, et al. Innovation in early medical education, no bells or whistles required. BMC Med Educ. 2020;20(1):39. doi:10.1186/s12909-020-1947-6

12. Qian Z-W, Huang G. Human capital and innovation ability in medical education: an empirical study. Eurasia J Math Sci Technol Educ. 2017;13 (8):5395-5403.

13. Shaw N. Medical education \& health informatics: time to join the 21st century? Stud Health Technol Inform. 2010;160(1):567-571.

14. Webster PC. Curricula reform needed to develop more tech-savvy physicians. CMAJ. 2011;183(10):E621-E622. doi:10.1503/cmaj.109-3913

15. Wartman SA, Combs CD. Medical education must move from the information age to the age of artificial intelligence. Acad Med. 2018;93:11071109. doi:10.1097/ACM.0000000000002044

16. Masters K. Artificial intelligence in medical education. Med Teach. 2019;41(9):976-980. doi:10.1080/0142159X.2019.1595557

17. Chan KS, Zary N. Applications and challenges of implementing artificial intelligence in medical education: integrative review. JMIR Med Educ. 2019;5:e13930. doi:10.2196/13930

18. Wood EA, Ange BL, Miller DD. Are we ready to integrate artificial intelligence literacy into medical school curriculum: students and faculty survey. J Med Educ Curr Dev. 2021;8:1-5. doi:10.1177/23821205211024078

\section{Publish your work in this journal}

Advances in Medical Education and Practice is an international, peer-reviewed, open access journal that aims to present and publish research on Medical Education covering medical, dental, nursing and allied health care professional education. The journal covers undergraduate education, postgraduate training and continuing medical education including emerging trends and innovative models linking education, research, and health care services. The manuscript management system is completely online and includes a very quick and fair peer-review system. Visit http://www.dovepress.com/testimonials.php to read real quotes from published authors.

Submit your manuscript here: http://www.dovepress.com/advances-in-medical-education-and-practice-journal 